\title{
Functionalizing Intraocular Microrobots with Surface Coatings
}

\author{
Olgaç Ergeneman, Jake J. Abbott, Görkem Dogangil, and Bradley J. Nelson
}

\begin{abstract}
Microrobots that can navigate bodily fluids will enable localized sensing and targeted drug delivery in parts of the body that are currently inaccessible or too invasive to access. Microrobots are typically envisioned as miniature mechatronic systems that utilize MEMS technology to incorporate sensing and actuation onboard. This paper presents a simpler alternative approach for the development of biomicrorobots consisting of magnetic platforms and functional coatings. Luminescence dyes immobilized in coatings can be excited and read wirelessly to detect analytes or physical properties. Drug coatings can be used for diffusion-based delivery, and may provide more efficient therapy than microsystems containing pumps, as diffusion dominates over advection at the microscale. Oxygen sensing for diagnosis in the eye and drug therapy for retinal vein occlusions are presented as example applications.
\end{abstract}

\section{INTRODUCTION}

During the past decade, the popularity of minimally invasive medical diagnosis and treatment has risen remarkably. Further advances in biomicrorobotics will enable the development of new diagnostic and therapeutic systems that provide major advantages over existing methods. Microrobots that can navigate bodily fluids will enable localized sensing and targeted drug delivery in parts of the body that are currently inaccessible or too invasive to access.

Microelectromechanical systems (MEMS) technology has enabled the integration of sensors, actuators, and electronics at microscales. In recent years, a great deal of progress has been made in the development of microdevices, and many devices have been proposed for different applications. However, placing these systems in a living body is limited by factors like biocompatibility, fouling, electric hazard, energy supply, and heat dissipation. In addition, the development of functional MEMS devices remains a time-consuming and costly process. Moving microsized objects in a fluid environment is also challenging, and a great deal of research has considered the development of microactuators for the locomotion of microrobots. However, to date the most promising methods for microrobot locomotion have utilized magnetic fields for wireless power and control, and this topic is now well understood [1]-[5]. A large number of micropumps have been developed for drug delivery, but as size is reduced diffusion begins to dominate over advection, making transport mechanisms behave differently at small scales. Consequently, future biomedical microrobots may differ from what is typically envisioned.

This work is supported by the NCCR Co-Me of the Swiss National Science Foundation.

The authors are with the Institute of Robotics and Intelligent Systems, ETH Zurich, 8092 Zurich, Switzerland. \{oergeneman, jabbott, goerkemd, bnelson\}@ethz.ch

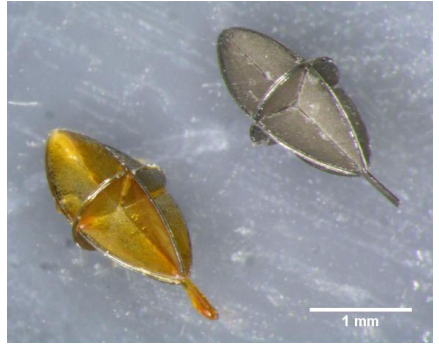

Fig. 1. Microrobot utilizing a functional coating. (Right) A bare magnetic microrobot made of thin assembled nickel pieces, based on [3]. (Left) A microrobot coated with an oxygen-sensitive film.

This paper presents an alternative approach for the development of biomicrorobots utilizing a magnetic platform and functional coatings for remote sensing and targeted drug delivery (Fig. 1). Coatings possessing sensor properties or carrying drugs may be superior to more complicated electromechanical systems. Luminescence dyes immobilized in coatings can be excited and read out wirelessly for detecting analytes or physical properties. Drugs coated on a carrier can be used for diffusion-based delivery and may provide more efficient therapy than microsystems containing pumps. Because of the discrepancy in scaling of volume and surface area, reservoirs built inside microfabricated devices may be insufficient, whereas surface coatings alone may provide sufficient volume. Fabrication of devices utilizing coatings will also be simple compared to systems with many electrical or mechanical components. All of these properties make wireless microrobots consisting of magnetic bodies and functional coatings feasible in the near term.

This work focuses on ophthalmic microrobots. The proposed devices can be inserted through a small incision in the sclera, and then control within the eye can be accomplished via applied magnetic fields. The eye is unique in that it is possible to observe the vasculature and visually track the microrobot through the pupil [6]. Throughout this paper, we consider the assembled-MEMS design shown in Fig. 1 , but the conclusions extend to other microrobot designs. The applications of biomicrorobots are also not limited to ophthalmology; the concepts described here are applicable to systems operating in other locations in the body.

The philosophy of designing simple structures with no actuation or intelligence onboard begs the question: Are these devices microrobots? It may be more accurate to think of these devices as end-effectors of novel manipulators where magnetic fields replace mechanical links, sensing is performed wirelessly, and system intelligence is located outside of the patient. However, this matter of semantics is 
inconsequential if the goal is to develop functional biomedical microdevices.

\section{Coatings for Remote Sensing}

Surface coatings can be used to fabricate minimally invasive wireless sensor devices, such as the intraocular sensor depicted in Fig. 2. The proposed device consists of a luminescence sensor film that is integrated with a magnetically controlled platform. This system can be used to obtain concentration maps of clinically relevant species (e.g., oxygen, glucose, urea, drugs) or physiological parameters (e.g., pressure, $\mathrm{pH}$, temperature) inside the eye, specifically in the preretinal area. Effects of specific physiological conditions on ophthalmic disorders can be conveyed.

These devices can also be used in the study of pharmacokinetics as well as the development of new drug delivery mechanisms, as summarized below [7]. The study of pharmacokinetics of drugs that diffuse into the eye following intraocular drug injection requires analysis of ocular specimens as they change in time. The risk of iatrogenic complications when penetrating into the ocular cavity with a needle has restricted ocular pharmacokinetic studies on animals and humans. Microdialysis has become an important method for obtaining intraocular pharmacokinetic data and it reduced the number of animals needed to estimate ocular pharmacokinetic parameter values. However the insertion of the probe and anesthesia have been shown to alter the pharmacokinetics of drugs. The microrobotic system presented in this paper can replace microdialysis probes for obtaining intraocular pharmacokinetic data as it provides an minimally invasive alternative for in vivo measurements of certain anayltes. Concentration as a function of time and position can be obtained by steering the magnetic sensor inside the vitreous cavity. Knowledge of concentration variations within the vitreous will expedite the optimization of drug administration techniques for posterior segment diseases.

\section{A. Luminescence Sensing}

Photoluminescence is the emission of electromagnetic radiation (i.e., photons) from a material in response to absorption of photons. The intensity and the lifetime of emission can be decreased by a variety of processes referred to as luminescence quenching. Optical luminescence sensors work based on quenching of luminescence in the presence of a quencher (i.e., analyte of interest); the decrease in luminescence is related to the quantity of the quencher. A number of devices using this principle have been demonstrated and the basic principles of different methods can be found in [8]. The quenching of luminescence is described by Stern-Volmer equations:

$$
\begin{aligned}
& \frac{I_{0}}{I}=1+K[Q] \\
& \frac{\tau_{0}}{\tau}=1+K[Q]
\end{aligned}
$$

where $I_{0}$ and $I$ are the luminescence intensities in the absence and in the presence of quencher, respectively, $\tau_{0}$ and

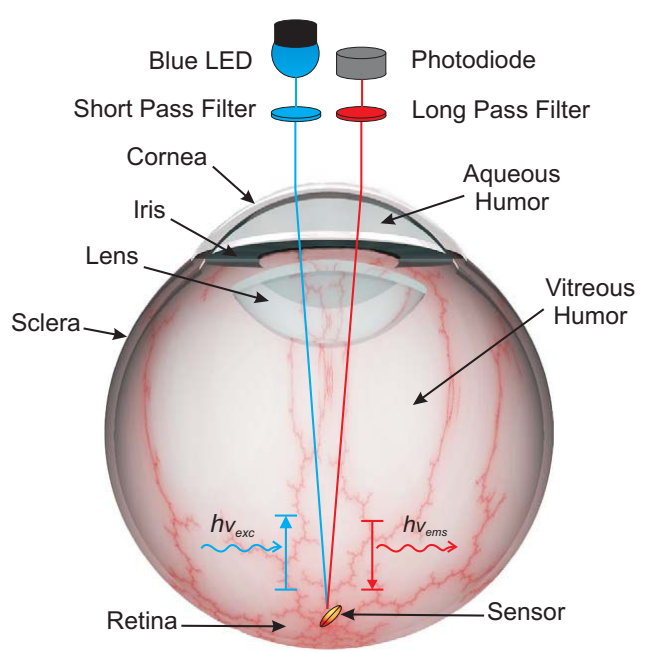

Fig. 2. Artist's conception of the magnetically controlled wireless sensor in the eye.

$\tau$ are the luminescence lifetimes in the absence and presence of quencher, respectively, $[Q]$ is the quencher concentration, and $K$ is the Stern-Volmer quenching constant whose units are the reciprocal of the units of $[Q]$. Luminescence dyes with high quantum yield, large dynamic range, and large Stokes shift are preferred for luminescence sensors. To be used as a sensor, these dyes need to be immobilized. They are usually bound to transparent and quencher-permeable supporting matrices such as polymers, silica gels, or solgels. Quencher permeability, selectivity, and the luminophore solubility are the important factors for choosing appropriate supporting matrices.

Luminescence sensing can be done either based on luminescence intensity or luminescence lifetime. The main difference between the two methods is that intensity is an extrinsic property whereas lifetime is an intrinsic property. Extrinsic techniques depend on parameters such as the dye concentration, optical surface quality, photo-bleaching, and incidence angle, which change from sample to sample. When the sensor's position changes, the optical path distance (OPD) from the light source to the sensor and back to the photo detector changes. The total amount of light collected by the sensor changes depending on the OPD and orientation. These quantities are hard to control in such a wireless sensor application, limiting the accuracy of this technique. Intrinsic properties do not depend on the parameters described above, making lifetime measurements more promising for wireless microrobotic applications.

There are two methods that are used for measuring luminescence lifetimes: time-domain measurements and frequency-domain measurements. In time-domain measurements the sample is excited with light pulses, and the intensity signal that changes as a function of time is measured and analyzed. In frequency-domain measurements the sample is excited with a periodic signal that consequently causes a modulated luminescence emission at the identical frequency. Because of the lifetime of emission, the emission signal has 


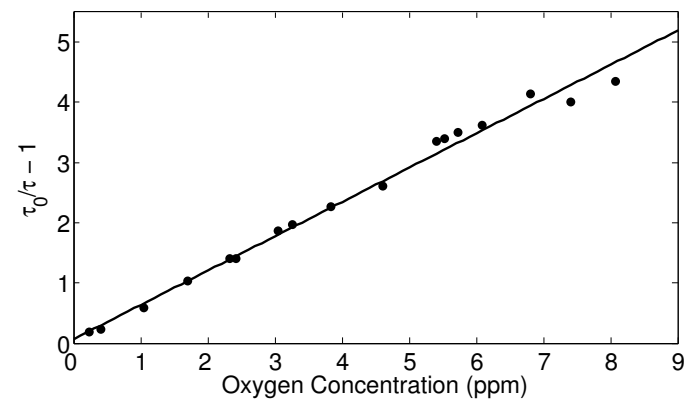

Fig. 3. Stern-Volmer plot of the N948 dyes immobilized in polystyrene film under various oxygen concentrations.

a phase shift with respect to the excitation signal. The input excitation signal is used as a reference to establish a zerophase position and the lifetime is obtained by measuring the phase shift between the excitation and emission signals.

\section{B. An Intraocular Oxygen Sensor}

The retina needs sufficient supply of oxygen and other nutrients to perform its primary visual function. Inadequate oxygen supply (i.e., retinal hypoxia) is correlated with major eye diseases including diabetic retinopathy, glaucoma, retinopathy of prematurity, age-related macular degeneration, and retinal vein occlusions [9]. Retinal hypoxia is presumed to initiate angiogenesis, which is a major cause of blindness in developed countries [10]. Attempts to test this hypothesis suffer from the current methods of highly invasive oxygen electrodes. Hypoxia is typically present at the end stages of retinal diseases. However, during the early stages, the relation between blood flow sufficiency, vessel patency, and tissue hypoxia are still unknown [11].The influence of oxygen on these diseases is not well understood and the ability to make long-term, non-invasive, in vivo oxygen measurements in the human eye is essential for better diagnosis and treatment. Measuring the oxygen tensions both in aqueous humor and vitreous humor, and particularly in the preretinal area, is of great interest in ophthalmic research.

To address these issues, an intraocular optical oxygen sensor utilizing a luminescence coating has been developed [12]. The sensor works based on quenching of luminescence in the presence of oxygen. A novel iridium phosphorescent complex $\mathrm{N} 948$ is designed and synthesized to be used as the oxygen probe. The main advantages of N948 iridium complex, when compared to other metal complexes, are its higher luminescence quantum yield, higher photo-stability, longer lifetime, stronger absorption band in the visible region, and larger Stokes shift. Polystyrene is chosen as the supporting matrix because of its high oxygen permeability and biocompatible nature. The microrobots are dip-coated with polystyrene film containing N948 dye, and good uniformity is achieved across the magnetic body. Biocompatibility tests must still be performed on the polysyrene film with embedded dye. If needed, an additional layer of pure polystyrene could be added to isolate the sensing layer.

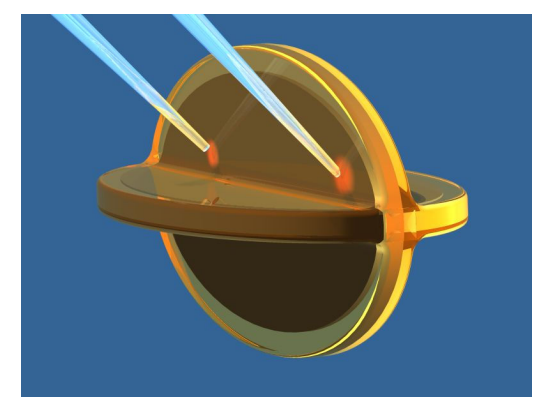

Fig. 4. Measurement of local gradients is possible exciting and reading out from different parts of the microrobot.

An experimental setup has been built to characterize the oxygen sensitivity of the sensor. The details of the sensor and characterization setup can be found in [12]. A blue LED is used as the excitation source for the oxygen sensor system and a photodiode is used to detect the luminescence. Optical filters are used to separate the emission signal from the excitation signal. The frequency-domain lifetime measurement approach is used in this work. De-ionized water is used for the dissolved oxygen measurements. The sensor's location in the setup is maintained with a magnet. The distances between the components and the sensor are chosen considering the geometry of the eye. A range of oxygen concentrations is achieved by bubbling air or nitrogen gas. Nitrogen replaces oxygen molecules in the solution, and air provides oxygen. Consequently, by applying nitrogen the oxygen concentration can be reduced, and by applying air it can be increased up to air saturation level at a given pressure and temperature condition. A commercial electrochemical oxygen sensor (Oxi340i, WTW $\mathrm{GmbH}$ ) is used as a reference; it was calibrated at the start of each experiment using its calibration vessel. The lowest and highest oxygen concentration levels achieved were $0.25 \mathrm{ppm}$ and $8.27 \mathrm{ppm}$, respectively. Three cycles were made going from the lowest concentration level to the highest and back to the lowest again. Seventeen measurements were taken at different oxygen concentration levels without the presence of ambient light. Figure 3 shows the SternVolmer plot as a function of oxygen concentration. As seen in this figure, a linear model proved to be an excellent predictor $\left(R^{2}=0.989\right)$ for oxygen concentrations compared to the commercial sensor. The empirically determined SternVolmer constant is $K=0.567 \mathrm{ppm}^{-1}$.

\section{Measuring Gradients}

It may be desirable to measure spatial gradients in a quantity. This can be accomplished by taking measurements while moving the microrobot. However, these measurements will be separated in time, and the movement of the microrobot could potentially affect the environment, particularly in a low-Reynolds-number regime. It is possible to measure gradients directly with a stationary microrobot. Specific locations on the microrobot can be excited and sensed simultaneously, as depicted in Fig. 4. This requires the ability to focus the excitation signal on a specific region of the microrobot. Clearly, this necessitates a greater level of 
sensing spatial resolution. Alternatively, multiple dyes with different emission spectra can be excited simultaneously, and the emitted signals can be band-pass filtered.

\section{CoAtings FOR TARgeted DRUg Delivery}

The main challenge of ophthalmic drug delivery is to keep desired drug concentrations in the target area for the desired duration, while minimizing the drug levels in the remainder of the body. To date, a variety of drug delivery approaches have been shown to be effective therapeutically. Some salient findings from [7] are summarized below. Ocular delivery can be achieved by topical administration, systemic administration, periocular injections, and intraocular injections. Depending on the target area, drug delivery can be achieved by penetrating through the cornea, conjunctiva, or sclera following topical administration (i.e., eye drops), or across blood-aqueous barrier along with blood-retinal barrier following systemic administration. Only a minute fraction of applied dose reaches the intraocular target area after topical and systemic administration. Drug delivery using gelatin wafers, collagen shields, and soft contact lenses placed on the cornea or in the cul-de-sac have been tested, as well as methods like iontophoresis. Drug delivery for posteriorsegment disorders (e.g., diabetic retinopathy, macular degeneration, retinal edema, retinal vein occlusions) has always been a challenge as it requires access to the retina and the choroid. Periocular injections and intraocular injections place the drug closer to the target tissue, overcoming some of the ocular barriers. Many of the drugs used to treat vitreous and retinal disorders have a narrow concentration range in which they are effective, and they may be toxic at higher concentrations. Slight changes in injection conditions (e.g., position, shape) will produce different drug concentrations within the vitreous, and therefore the efficacy of the treatment produced by the drug can be sensitive to injection conditions. Intraocular injections have also been associated with serious side effects, such as endophthalmitis, cataract, hemorrhage, and retinal detachment [13], and long-duration drug delivery is not possible with these methods.

New drug-delivery methods provide many advantages compared to the traditional methods. However, it is not always possible to deliver drugs to the target tissue with existing methods. Superior methods for targeted drug delivery are needed, and robotic assistance in drug delivery will have major benefits. Devices inserted into the aqueous or vitreous cavity bear great potential for drug delivery. Recently, methods to deliver drugs using carriers such as liposomes, gels, and nanoparticles have been evaluated. Methods to achieve desired concentrations over long periods of time using drugs that become active inside the eye (prodrugs) are also being investigated. Controlled-release devices and biodegradable implants can increase the effectiveness of these devices.

At the microscale, diffusion becomes the dominant mechanism for mass transport. Low-Reynolds-number flow is laminar, and the lack of turbulent mixing puts a diffusion limit on drug delivery using micropumps. We propose an

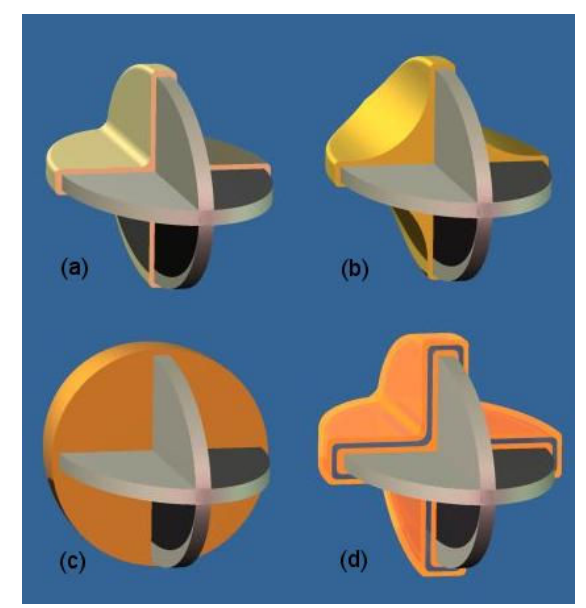

Fig. 5. Drug coatings can range from thin surface coatings to coatings that take advantage of the total available volume created by the microrobot structure. Coatings are shown only at the back part of the microrobot.

alternative approach to targeted drug delivery: wireless magnetic microrobots surface coated with drug. The microrobot will be steered to the site of action as it is tracked visually through the pupil, and it will be kept at this position as the drug is released from the microrobot by diffusion.

\section{A. Quantity of Coated Drug}

Carrying drug by surface coating becomes more desirable as size is reduced (Fig. 5). Consider an assembled microrobot like those shown in Fig. 1. The microrobot can be modeled by two elliptical pieces of magnetic material of length $2 a$, width $2 b$, and thickness $c$, and by a circular piece of diameter $2 b$ and thickness $c$. The volume of the magnetic structure is calculated as

$$
v_{s}=\left(2 \pi a b+\pi b^{2}\right) c-(2 a+4 b) c^{2}+c^{3}
$$

The microrobot has a volumetric footprint of an ellipsoid of volume

$$
v_{e}=\frac{4}{3} \pi a b^{2}
$$

If we consider a coating of drug that fills in the entire ellipsoidal volumetric footprint of the microrobot (similar to Fig. 5(c)), the volume of drug carried is simply the volume of the ellipsoid minus the volume of the magnetic structure

$$
v_{f}=v_{e}-v_{m}
$$

If we consider a single thin surface coating of thickness $t$, (similar to Fig. 5(a)), the volume of drug carried is given by

$$
v_{t}=\left(4 \pi a b+2 \pi b^{2}\right) t-(8 a+16 b-12 c) t^{2}+8 t^{3}
$$

Figure 6 shows the effect of scaling on the ability to carry drug by surface coating. For even relatively large microrobots, the amount of drug carried on the surface with a single thin coating is comparable to the total volume of the magnetic structure. As the size of the microrobot is reduced, the volume of drug in a single thin coating becomes comparable to the total ellipsoidal volume of the microrobot. In practice, any fabricated reservoir could only amount to a 


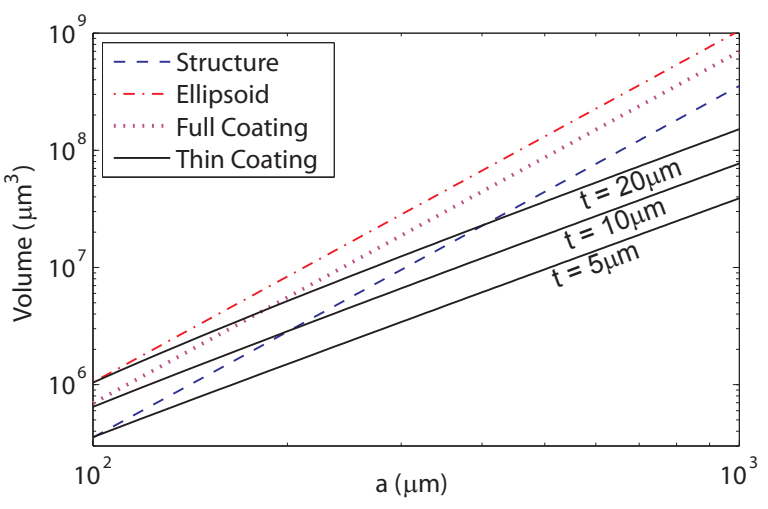

Fig. 6. Effect of scaling on volume with microrobots of the type shown in Fig. 1. Curves correspond to (3)-(6), respectively, with $b=a / 2$ and $c=a / 10$.

fraction of the total volume of the structure, and the drug would need to be in solution (that is, diluted) in order to be pumped. The ability to surface coat highly concentrated drug increases the benefits of surface coatings even beyond what is observed in Fig. 6.

In order to bind more proteins or drugs onto a microrobot of the same surface area, multilayer surface coatings or coatings embedded in different base matrices should be developed (Fig. 5(d)). Among others, hydrogels, agarose, starch microcapsulations, polymer matrices, liposomes, and biodegradable needles are widely used for making drug delivery matrices that can hold much more drug due to their material properties [14], [15]. These materials can be used to encapsulate drug molecules as an outer coating, enabling multilayer coatings. These multilayer coatings can be used to coat multiple drug types on one microrobot, or used to fine tune delivery times or dosage. Alternatively, embedding drug molecules in a porous matrix facilitates slower diffusion and more drug loading capacity. Controlled release of drugs has been demonstrated using intelligent polymers that respond to stimuli such magnetic fields, ultrasound, temperature, and $\mathrm{pH}$. They enable fine tuning of diffusive drug release.

\section{B. Drug Delivery for Retinal Vein Occlusions}

Retinal vein occlusion (RVO) is a common retinovascular disease caused by obstruction of blood flow due to clot formation. RVO is among the most common causes of vision loss around the world, with one study reporting a prevalence of $1.6 \%$ in adults aged 49 years or older [16]. Various treatment methods for RVO have been proposed and attempted, however to date there is no effective clinical treatment for RVO. Among these methods, prolonged local intravenous thrombolysis (i.e., clot dissolution) with tissue plasminogen activator (t-PA) injection is the most promising treatment [17], based on excessive postoperative complications or inconclusive clinical trials of other methods.

Retinal drug delivery by injections requires precise manipulation that is constrained by the limits of human performance and perception [18]. Retinal veins are small delicate structures surrounded by fragile retinal tissue, and prolonged manual cannulation of retinal veins risks causing permanent damage to the retina. Robotic systems have been proposed to assist with retinal vein cannulation, utilizing robot-assisted surgical instruments that pass through a hole in the sclera as in conventional vitreoretinal surgery [19], [20].

We propose an alternative approach to RVO treatment: a wireless magnetic microrobot coated with clot-dissolving tPA. The microrobot will be steered to the thrombus site as it is tracked visually through the pupil, and will be immobilized in close proximity of the retinal veins. Immobilization can be achieved by puncturing and docking to a retinal vein [21]. Diffusion of t-PA from the surface coating of the microrobot into the clotted region will start clot dissolution. There is strong evidence that t-PA in the preretinal area can diffuse into the retinal vasculature and break clots [22]. Since t$\mathrm{PA}$ is an enzyme, and the clot dissolution reaction rate depends on enzyme reaction rate, long-term release of t-PA is thought to be more effective than bolus injections [23]. The proposed delivery mechanism provides drug release without the need for a micropump, and an efficient therapy using small amounts of t-PA over prolonged periods. Moreover, a microrobot is potentially less invasive than other methods, and has the potential to be left in the eye for extended periods of time, even in an outpatient scenario. However, it is not yet known what quantity of t-Pa is required to effectively dissolve a clot using the proposed method.

\section{Preliminary Drug Release Experiments}

This section presents the results of preliminary drug release experiments using the untethered microrobot and discusses the feasibility of microrobotic drug delivery. A drug substitute is coated on microrobots, the release kinetics is characterized, and the amount of drug that can be coated in a single layer on a microrobot is quantified.

In order to analyze the release kinetics of a diffusionbased drug delivery microrobot, in vitro experiments are conducted. As the drug molecule substitute, bovine serum albumin (BSA) was chosen. BSA is a plasma protein that can be used as a blocking agent or added to diluents in numerous biochemical applications. BSA is used because of its stability, its inert nature in many biochemical reactions, its representative molecular size, and its low cost.

Four elliptical microrobot pieces of length $900 \mu \mathrm{m}$, width $450 \mu \mathrm{m}$, and thickness $50 \mu \mathrm{m}$ are used as the magnetic platform holding the coating. The pieces are made from electroplated nickel and then coated with titanium for biocompatibility as described in [21]. The pieces are sterilized in ethanol and then washed with sterilized DI-water. Microrobot pieces are placed in different wells of a 96-well culture plate. A sterilized BSA-solution of $3 \mathrm{mg} / \mathrm{mL}$ is prepared and labeled with Alexa-Fluor-546 (Molecular Probes) fluorescent marker. This solution is then mixed with sterilized PBS in order to create solutions with different concentrations of labeled-BSA molecules. Three of the microrobot pieces are dipped in BSA concentrations of $3 \mathrm{mg} / \mathrm{mL}, 2 \mathrm{mg} / \mathrm{mL}$, $1 \mathrm{mg} / \mathrm{mL}$, respectively, and one is dipped into a pure PBS solution, which contained no BSA, as a control set. The 


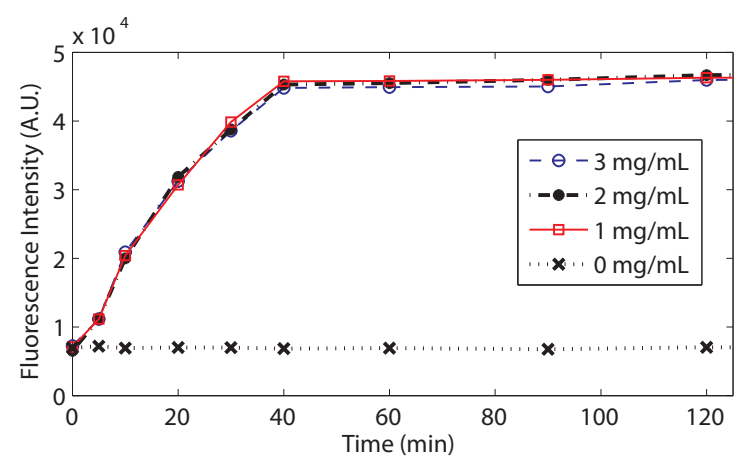

Fig. 7. Fluorescence intensity vs. time for the release experiment.

pieces are left in the solutions to allow the BSA to bind to the microrobot. The surface-coating process is done for 12 hours at room temperature in a humidity chamber.

Coated microrobot pieces are taken from coating wells and placed in new wells filled with $200 \mu \mathrm{L}$ PBS each. Following that, the florescence intensity is measured in set time intervals for three days using an automated spectrum analyzer (Tecan Infinite 200 Multiwell Plate Reader). In this way, the kinetics of diffusion-based drug delivery with surface-coated microrobots are obtained.

Fig. 7 quantifies the amount of time required to release the drug through diffusion, and it also gives qualitative information about the kinetics of release. It is clear that the concentration of the coating solution does not affect the amount of drug bound to the surface. This provides strong evidence that the amount of drug will be limited by the surface area of the microrobot.

Next, the amount of BSA released from a single piece is quantified. The release wells of the culture plates are analyzed in the multiwell plate reader for fluorescence and absorbance values. The BSA standard concentration curve is obtained by preparing a Bradford Assay with ten different known concentrations of BSA in 1:2 dilutions, and analyzing this assay for fluorescence and absorbance. The obtained standard curve is used to calibrate the multiwell plate reader. The fluorescence intensity in the release wells is measured and, using the calibration curve, the amount of BSA released is found to be $2.5 \pm 0.1 \mu \mathrm{g}$.

\section{CONCLUSIONS AND OUTLOOK}

We have demonstrated how surface coatings can be used to functionalize biomedical microrobots that make use of magnetic fields for wireless power and control. By taking this simple approach to design and fabrication, it may be possible to create clinical microrobots in the near term. We presented intraocular oxygen sensing and drug delivery for retinal vein occlusions as two potential applications. There are also promising new therapies like gene therapy, where there is a need to deliver polypeptide medications and DNA inhibitors, which can take advantage of new posterior-segment drug delivery devices. It is also possible to incorporate multiple functions on a single device by coating individual components of the microrobot before assembly.

\section{REFERENCES}

[1] J. J. Abbott, O. Ergeneman, M. P. Kummer, A. M. Hirt, and B. J. Nelson, "Modeling magnetic torque and force for controlled manipulation of soft-magnetic bodies," IEEE Trans. Robotics, vol. 23, no. 6, pp. 1247-1252, 2007.

[2] Z. Nagy, O. Ergeneman, J. J. Abbott, M. Hutter, A. M. Hirt, and B. J. Nelson, "Modeling assembled-MEMS microrobots for wireless magnetic control," in Proc. IEEE Int. Conf. Robotics and Automation, May 2008.

[3] K. B. Yesin, K. Vollmers, and B. J. Nelson, "Modeling and control of untethered biomicrorobots in a fluidic environment using electromagnetic fields," Int. J. Robotics Research, vol. 25, no. 5-6, pp. 527-536, 2006.

[4] K. Ishiyama, K. I. Arai, M. Sendoh, and A. Yamazaki, "Spiral-type micro-machine for medical applications," J. of Micromechatronics, vol. 2, no. 1, pp. 77-86, 2003.

[5] G. T. Gillies, R. C. Ritter, W. C. Broaddus, M. S. Grady, M. A. Howard III, and R. G. McNeil, "Magnetic manipulation instrumentation for medical physics research," Review of Scientific Instruments, vol. 65 , no. 3, pp. 533-562, 1994.

[6] C. Bergeles, K. Shamaei, J. J. Abbott, and B. J. Nelson, "On imaging and localizing untethered intraocular devices with a stationary camera," in Proc. IEEE Int. Conf. on Biomedical Robotics and Biomechatronics, 2008.

[7] A. K. Mitra, Ophthalmic Drug Delivery Systems. New York: Marcel Dekker Inc., 2003.

[8] J. R. Lakowicz, Principles of Fluorescence Spectroscopy, 2nd ed. Kluwer Academic/Plenum Publishers, 1999.

[9] N. R. Galloway, W. M. K. Amoaku, P. H. Galloway, and A. C. Browning, Common Eye Diseases and their Management, 3rd ed. London: Springer, 2005.

[10] B. Berkowitz and C. Wilson, "Quantitative mapping of ocular oxygenation using magnetic resonance imaging," Magn. Reson. Med., vol. 33, no. 4, pp. 579-581, 1995.

[11] R. Shonat and A. Kight, "Oxygen tension imaging in the mouse retina," Annals of Biomedical Eng., vol. 31, pp. 1084-1096, 2003.

[12] O. Ergeneman, G. Dogangil, M. P. Kummer, J. J. Abbott, M. K. Nazeeruddin, and B. J. Nelson, "A magnetically controlled wireless optical oxygen sensor for intraocular measurements," IEEE Sensors Journal, vol. 8, no. 1, pp. pp. 29-37, 2008.

[13] D. H. Geroski and H. F. Edelhauser, "Drug delivery for posterior segment eye disease," Invest. Ophthalmol. Vis. Sci., vol. 41, no. 5, pp. 961-964, 2000.

[14] X. Cao, S. Lai, and L. J. Lee, "Design of a self-regulated drug delivery device," Biomedical Microdevices, vol. 3, no. 2, pp. 109-118, 2001.

[15] J.-H. Park, M. G. Allen, and M. R. Prausnitz, "Polymer microneedles for controlled-release drug delivery," Pharm. Research, vol. 23, no. 5, pp. 1008-1018, 2006.

[16] W. M. Tang and D. P. Han, "A study of surgical approaches to retinal vascular occlusions," Arch. Ophthalmol., vol. 118, pp. 138-143, 2000.

[17] H. Shahid, P. Hossain, and W. M. Amoaku, "The management of retinal vein occlusion: Is interventional ophthalmology the way forward?" British J. Ophthalmol., vol. 90, pp. 627-639, 2006.

[18] A. D. Jagtap and C. N. Riviere, "Applied force during vitreoretinal microsurgery with handheld instruments," in Proc. IEEE Int. Conf. Engineering in Medicine and Biology Society, 2004, pp. 2771-2773.

[19] B. Mitchell, J. Koo, I. Iordachita, P. Kazanzides, A. Kapoor, J. Handa, G. Hager, and R. Taylor, "Development and application of a new steady-hand manipulator for retinal surgery," in Proc. IEEE Int. Conf. Robotics and Automation, 2007, pp. 623-629.

[20] C. N. Riviere, W. T. Ang, and P. K. Khosla, "Toward active tremor canceling in handheld microsurgical instruments," IEEE Trans. on Robotics and Automation, vol. 19, no. 5, pp. 793-800, 2003.

[21] G. Dogangil, O. Ergeneman, J. J. Abbott, S. Pane, H. Hall, S. Muntwyler, and B. J. Nelson, "Toward targeted retinal drug delivery with wireless magnetic microrobots," in Proc. IEEE/RSJ Int. Conf. on Intelligent Robots and Systems, 2008.

[22] N. G. Ghazi, B. N. Noureddine, R. S. Haddad, F. A. Jurdi, and Z. F. Bashshur, "Intravitreal tissue plasminogen activator in the management of central retinal vein occlusion," Retina, vol. 23, pp. 780-784, 2003.

[23] M. K. Tameesh et al., "Retinal vein cannulation with prolonged infusion of tissue plasminogen activator (t-PA) for the treatment of experimental retinal vein occlusion in dogs," Am. J. Ophthalmol., vol. 138, no. 5, pp. 829-839, 2004. 